Slavica

bruxellensia

\section{Slavica bruxellensia}

Revue polyphonique de littérature, culture et histoire

slaves

$6 \mid 2010$

Linguistique russe

\title{
The Golden Age of Polish Philosophy : Kazimierz Twardowski's Philosophical Legacy, Lapointe S. (éd.)
}

\section{Sébastien Richard}

\section{OpenEdition}

\section{Journals}

Édition électronique

URL : http://journals.openedition.org/slavica/363

DOI : $10.4000 /$ slavica.363

ISSN : 2034-6395

\section{Éditeur}

Université libre de Bruxelles - ULB

\section{Édition imprimée}

Pagination : 67-68

ISSN : 2031-7654

\section{Référence électronique}

Sébastien Richard, «The Golden Age of Polish Philosophy : Kazimierz Twardowski's Philosophical Legacy, Lapointe S. (éd.) », Slavica bruxellensia [En ligne], 6 | 2010, mis en ligne le 15 juin 2010, consulté le 22 septembre 2020. URL : http://journals.openedition.org/slavica/363 ; DOI : https://doi.org/10.4000/ slavica.363

Ce document a été généré automatiquement le 22 septembre 2020.

\section{(i) $९$}

Les contenus de Slavica bruxellensia sont mis à disposition selon les termes de la Licence Creative Commons Attribution - Pas d'Utilisation Commerciale - Pas de Modification 3.0 France. 


\section{The Golden Age of Polish Philosophy: Kazimierz Twardowski's Philosophical Legacy, Lapointe S. (éd.)}

Sébastien Richard

\section{RÉFÉRENCE}

The Golden Age of Polish Philosophy : Kazimierz Twardowski's Philosophical Legacy, Lapointe S. (éd.), Dordrecht-Heidelberg-Londres-New York, Springer, 2009, 254 p. 
1 Les articles regroupés dans ce volume sont issus de deux colloques organisés en 2004 autour du thème de l'histoire de la philosophie polonaise: l'un s'est tenu à Montréal et l'autre à Paris. L'ouvrage est principalement consacré à la période de la philosophie polonaise qui s'étend de 1895 jusqu'à la Deuxième Guerre mondiale. Lors de cette dernière, de nombreux intellectuels polonais furent assassinés ou bien immigrèrent dans d'autres pays, mettant ainsi fin aux plus glorieuses années de la philosophie polonaise. Cet âge d'or est avant tout représenté par l'École de Lvov-Varsovie, à laquelle est presque entièrement dédié ce volume. La naissance de celle-ci remonte à la nomination de Kazimierz Twardowski, un ancien élève de Franz Brentano, comme professeur de philosophie à l'Université

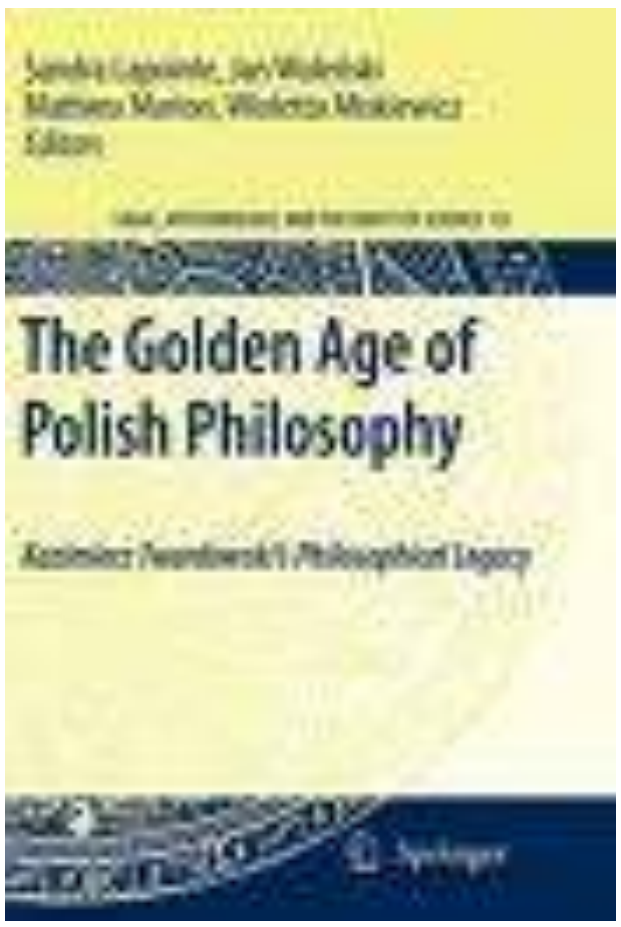
de Lvov en 1895. Son influence n'est pas tant due à ses écrits (Twardowski a peu publié), mais surtout à son enseignement et à sa vision de la philosophie. Celle-ci est à la fois analytique et brentanienne, et en ce sens l'École de Lvov-Varsovie peut être rattachée à ce qu'on appelle la «philosophie d'Europe centrale» ou "École de Brentano». Ainsi, la philosophie de l'École de Twardowski se caractérise par une attention particulière au langage, à la clarté et à la science, par un rejet du kantisme. De plus, elle n'exclut pas toute métaphysique et ancre ses recherches dans l'histoire de la philosophie, en particulier dans l'aristotélisme. Cette conception de la philosophie sera diffusée par la plupart des élèves de Twardowski qui occuperont différents postes académiques, d'abord à l'Université de Lvov, puis, à partir de 1918, à celle de Varsovie.

2 Cette deuxième branche de l'École de Lvov-Varsovie est surtout connue pour ses contributions à la logique et à la philosophie des mathématiques. Qu'il nous suffise de citer les noms de Jan Łukasiewicz, Stanisław Leśniewski, Alfred Tarski, Tadeusz Kotarbiński et Kazimierz Ajdukiewicz, et l'on aura un aperçu de la contribution majeure apportée dans ce domaine par cette école. De plus, ces découvertes ne resteront pas confinées en Pologne et seront largement diffusées après la Deuxième Guerre mondiale, principalement dans le monde académique anglo-saxon.

3 L'ouvrage ne se consacre pas seulement à ces aspects bien connus de la philosophie polonaise, mais également à la contribution au problème corps-esprit de Henryk Mehlberg, à la phénoménologie analytique de Leopold Blaustein, au thomisme analytique du Cercle de Cracovie, en la personne de Jan Salamucha, ainsi qu'aux importantes études d'histoire de la philosophie médiévale de Krzysztof Michalski.

4 L'un des intérêts de ce volume est donc d'offrir une perspective large sur cette période particulière de la philosophie polonaise, en ne se limitant pas à l'héritage de Twardowski et à l'École de Lvov-Varsovie, lesquels ont déjà fait l'objet de nombreuses études, notamment de la part de Jan Woleński. Les articles sont regroupés en quatre 
grandes sections: "Twardowski et la philosophie scientifique polonaise", "philosophie de la logique et des mathématiques", «la philosophie polonaise de l'esprit » et « autour de l'École de Twardowski ».

5 L'ouvrage offre un bel échantillon des recherches dans ces différents domaines. Les articles sont écrits par des spécialistes internationaux reconnus de l'âge d'or de la philosophie polonaise. On pourra parfois regretter le manque d'originalité de quelques articles retenus, certains ayant déjà été publiés ailleurs, le thème de quelques autres ayant déjà fait l'objet d'études plus approfondies. Mais, l'ensemble constitue tout de même une belle introduction à la période phare de la philosophie polonaise. Le tout est précédé d'une utile introduction historique à la philosophie polonaise depuis le XII siècle jusqu’à la Deuxième Guerre mondiale.

\section{INDEX}

Index géographique : Pologne, Ukraine

Index chronologique : entre-deux-guerres, XXe siècle

Mots-clés : école de Lvov-Varsovie, philosophie polonaise

\section{AUTEURS}

\section{SÉBASTIEN RICHARD}

Aspirant du F.N.R.S. Université Libre de Bruxelles 